\title{
Preoperative and Postoperative Bone Mineral Density Change and Risk Factor Analysis in Patients with a GH-Secreting Pituitary Adenoma
}

\author{
Li'nan Qin $\mathbb{i D}^{1,},{ }^{1,2,3}$ Xiaopeng Guo $\mathbb{i D}^{1,2,3}$ Lu Gao ${ }^{(i)}{ }^{1,2,3}$ Zihao Wang, ${ }^{1,2,3}$ Chenzhe Feng, ${ }^{1,2,3}$ \\ Kan Deng $\mathbb{1},^{1,2,3}$ Wei Lian $\left(\mathbb{1},,^{1,2,3}\right.$ and Bing Xing $\mathbb{1}^{1,2,3}$ \\ ${ }^{1}$ Department of Neurosurgery, Peking Union Medical College Hospital, \\ Chinese Academy of Medical Sciences and Peking Union Medical College, Beijing 100730, China \\ ${ }^{2}$ China Pituitary Disease Registry Center, Chinese Pituitary Adenoma Cooperative Group, Beijing 100730, China \\ ${ }^{3}$ Key Laboratory of Endocrinology of National Health and Family Planning Commission, Beijing 100730, China
}

Correspondence should be addressed to Bing Xing; xingbingemail@aliyun.com

Received 6 August 2019; Revised 5 October 2019; Accepted 16 October 2019; Published 3 November 2019

Academic Editor: Marek Bolanowski

Copyright (C) 2019 Li'nan Qin et al. This is an open access article distributed under the Creative Commons Attribution License, which permits unrestricted use, distribution, and reproduction in any medium, provided the original work is properly cited.

Purpose. This study analysed changes in bone mineral density (BMD) at different sites in patients with acromegaly and postoperative BMD changes and explored risk factors associated with BMD. Methods. Clinical data of 39 patients with growth hormone- (GH-) secreting pituitary adenomas and 29 patients with nonfunctioning pituitary adenomas who were newly diagnosed in neurosurgery from January 2016 to December 2018 were retrospectively analysed, including measurements of preoperative and postoperative BMD, serum GH glucose inhibition, random GH and IGF-1, and other anterior pituitary hormones. Results. The average patient age and disease duration were 43.74 (33.41-54.07) years and 72.15 (22.82-121.48) months, respectively. Compared with patients with nonfunctioning adenomas, patients with GH-secreting pituitary adenomas had significantly higher BMDs at L1, L2, femoral neck, Ward triangle, trochanter, femoral shaft, and total hip sites $(p<0.05)$. The BMD $Z$ score at L1 and femoral neck sites significantly increased $(p<0.05)$. Thirteen patients underwent re-examination of BMD 1 year postsurgery, and the BMD $Z$ score was reduced to normal levels at L1, L2, L3, L4, L1-L4, and L2-L4 compared with preoperative levels $(p<0.05)$. Postoperative BMD $Z$ scores in the femoral neck and total hip were significantly increased $(p<0.05)$. Disease duration was negatively correlated with the lumbar-spine BMD $Z$ score. IGF-1 burden was negatively correlated with the BMD $Z$ score at L1 and L1-L4. Multiple regression analysis showed that IGF-1 burden was a risk factor for a BMD $Z$ score decrease at L1 and L1-L4. Conclusion. BMD in patients with GH-secreting pituitary adenomas (compared with nonfunctional adenomas) increased at L1, L2, femoral neck, Ward triangle, trochanter, femoral shaft, and total hip sites. Lumbarspine BMD $Z$ score recovered to normal levels postsurgically when GH and IGF-1 levels were controlled. BMD $Z$ score was negatively correlated with disease duration and IGF-1 burden in patients with GH-secreting pituitary adenomas, and IGF-1 burden was an independent risk factor for reduced lumbar-spine BMD $Z$ score.

\section{Introduction}

Acromegaly is an endocrine and metabolic disorder syndrome caused by the oversecretion of growth hormone (GH) and insulin-like growth factor-1 (IGF-1), which results in excessive growth of bones, soft tissues, and internal organs. The structure and function of bones in patients with acromegaly are affected [1]. Among patients with acromegaly,
$58 \%$ have osteoporosis of the lumbar spine, $74 \%$ have osteoporosis of the femoral neck, and the incidence of vertebral fracture is approximately $39 \%$ [2]. More than $95 \%$ of acromegaly cases are caused by a GH-secreting pituitary adenoma [3]. GH and IGF-1 have anabolic effects on bone metabolism that can lead to increased bone formation and resorption [4]. However, the changes in bone mineral density (BMD) in patients with acromegaly have been 
controversial in the past few decades. Most studies have shown that patients have increased BMD, and some studies have revealed no significant changes; however, a few studies have reported decreased bone mineral density [5-8]. We suppose that, after surgery, the BMD should change, as the elevated GH and IGF-1 levels are controlled. To date, there is a lack of conclusive evidence that the BMD in patients with acromegaly decreases after surgery. Therefore, we aimed to investigate the preoperative $\mathrm{BMD}$ and to determine the postoperative BMD changes in patients with acromegaly in the Chinese population.

In addition, $\mathrm{GH}$ mediates bone metabolism through IGF-1, which is produced in the liver [9]. The IGF-1 level is considered positively correlated with BMD in patients with acromegaly [10-12]. The effect of IGF-1 on cortical bone seems to be more dominant than that on trabecular bone in mouse models [13]. Therefore, we aimed to examine the correlation between IGF-1 levels and BMD in humans and to explore other factors that affect BMD in patients with acromegaly.

The purpose of this study was to investigate the preoperative $\mathrm{BMD}$, to analyse the effect of acromegaly cure on BMD through successful surgery, and to explore risk factors associated with BMD in patients with acromegaly.

\section{Materials and Methods}

2.1. Study Population. Patients first diagnosed with a GHsecreting pituitary adenoma or nonfunctioning pituitary adenoma at Peking Union Medical College Hospital (PUMCH) from January 2016 to December 2018 were studied. The inclusion criteria were as follows: (1) endocrine diagnostic criteria: elevated serum IGF-1 level and lack of suppression of $\mathrm{GH}$ to $<1 \mu \mathrm{g} / \mathrm{L}$ following documented hyperglycaemia during an oral glucose load [3]; (2) pituitary enhanced MRI confirming a space-occupying lesion in the sellar region; (3) typical clinical manifestations of acromegaly; (4) pathologically confirmed GH-secreting adenoma; (5) performance of a BMD examination through DXA; and (6) no history of medical treatment or radiation. Patients with a nonfunctioning pituitary adenoma were selected as the control group. The inclusion criteria of control group were as follows: (1) endocrine diagnostic criteria: without hormone oversecretion for each specific pituitary tumour type [14]; (2) clinically diagnosed with a nonfunctioning adenoma; and (3) performance of a BMD examination through DXA before surgery. The exclusion criteria were (1) diagnosis of neoplastic disease; (2) diagnosis of osteoporosis-related disease, such as PCOS, hyperthyroidism, and hyperparathyroidism; (3) use of drugs that affect BMD or bone metabolism; or (4) chronic liver or kidney diseases. Disease control was defined as random GH $<1 \mu \mathrm{g} / \mathrm{L}$ or nadir $\mathrm{GH}$ after OGTT $<0.4 \mu \mathrm{g} / \mathrm{L}$ and age-sex normalized IGF-1 [15].

Informed consent was obtained from each patient prior to enrolment. This study was approved by the Ethics Committee of PUMCH at the Chinese Academy of Medical Sciences and Peking Union Medical College.
2.2. Biochemical Measurement. The methods for hormone evaluation are described in our previously published article [16].

2.3. BMD. BMD was measured at the lumbar spine (L1-L4), femoral neck, Ward triangle, trochanter, femoral shaft, and total hip by dual-energy X-ray absorptiometry (Lunar Prodigy Advance, Lunar Corporation, Madison, WI, USA). The T-score is defined as the difference between the measured BMD and the bone peak in young people of the same sex. The $Z$ score is defined as the difference between the measured BMD and the average BMD of people of the same age and race. The BMD examination was performed after hospital admission but before the surgery or at least 3 months after surgery during clinical re-examination.

2.4. Statistical Analysis. All data are expressed as the mean \pm SD. Student's $T$ and Mann-Whitney's $U$ tests were used to compare the $\mathrm{GH}$-secreting pituitary adenoma group and the nonfunctional group depending on whether it was normally distributed. A paired $T$ test was used to compare the preoperative and postoperative group BMD values. Pearson's correlation coefficient or Spearman's rank order was assessed to determine the correlations between BMD and other parameters. Stepwise multiple linear regression was conducted to identify potential predictive factors for $\mathrm{BMD}$ at each site. Statistical significance was accepted with a $p$ value $<0.05$.

\section{Results}

3.1. Patient Characteristics. Thirty-nine patients with GHsecreting pituitary adenomas and 29 patients with nonfunctioning pituitary adenomas were included. Thirteen patients with $\mathrm{GH}$-secreting pituitary adenomas had controlled disease and underwent re-examination of BMD 1 year after surgery. Characteristics of the study population are shown in Table 1 . The age and body mass index (BMI) of the two groups were matched. The average disease duration of patients with $\mathrm{GH}$-secreting pituitary adenomas was 72.15 (22.82-121.48) months, and the average adenoma size was 15.97 (9.1-22.84) mm. GH and IGF-1 levels in patients with $\mathrm{GH}$-secreting pituitary adenomas were significantly higher than those in the control group (both $p$ values $<0.001$ ). In addition, FT3 (free triiodothyronine), FT4 (free thyroxine), and phosphorus levels were increased significantly $(p<0.05$, respectively).

\section{2. $B M D$}

3.2.1. BMD in the GH-Secreting Pituitary Adenoma Group and Nonfunctioning Adenoma Group. The BMD comparison between the $\mathrm{GH}$-secreting pituitary adenoma group and controls is shown in Table 2. In the $\mathrm{GH}$-secreting pituitary adenoma group, BMD was significantly elevated at the L1, L2, femoral neck, Ward triangle, trochanter, femoral shaft, and total hip sites compared with the corresponding values in patients with nonfunctioning adenomas $(p=0.006$, 
TABLE 1: Basic information of the GH-secreting pituitary adenoma group and the nonfunctioning pituitary adenoma group.

\begin{tabular}{|c|c|c|c|}
\hline & GH-secreting pituitary adenoma & Nonfunctioning pituitary adenoma & $p$ \\
\hline$N$ & 39 & 29 & - \\
\hline Gender $(\mathrm{M} / \mathrm{F})$ & $19 / 20$ & $15 / 14$ & - \\
\hline Age (years) & $43.74 \pm 10.33$ & $49.14 \pm 16.00$ & 0.120 \\
\hline BMI $\left(\mathrm{kg} / \mathrm{m}^{2}\right)$ & $26.03 \pm 4.57$ & $24.69 \pm 2.73$ & 0.292 \\
\hline Disease duration (months) & $72.15 \pm 49.33$ & $17.97 \pm 34.84$ & $<0.001$ \\
\hline Adenoma size $(\mathrm{mm})$ & $15.97 \pm 6.87$ & $26.43 \pm 9.63$ & $<0.001$ \\
\hline $\mathrm{GH}(\mathrm{ng} / \mathrm{mL})$ & $33.49 \pm 48.75$ & $0.80 \pm 1.77$ & $<0.001$ \\
\hline IGF-1 (ng/mL) & $855.00 \pm 261.91$ & $168.52 \pm 81.14$ & $<0.001$ \\
\hline T3 (ng/mL) & $1.37 \pm 1.47$ & $0.97 \pm 0.22$ & 0.109 \\
\hline $\mathrm{T} 4$ (ng/mL) & $7.64 \pm 1.70$ & $7.45 \pm 1.64$ & 0.660 \\
\hline FT3 $(\mathrm{pg} / \mathrm{mL})$ & $3.25 \pm 0.70$ & $2.65 \pm 0.45$ & $<0.001$ \\
\hline FT4 $(\mathrm{pg} / \mathrm{mL})$ & $1.17 \pm 0.21$ & $1.06 \pm 0.20$ & 0.041 \\
\hline TSH $(\mathrm{mU} / \mathrm{L})$ & $1.55 \pm 1.03$ & $2.33 \pm 2.59$ & 0.143 \\
\hline $\mathrm{F}(\mathrm{ng} / \mathrm{mL})$ & $10.12 \pm 5.22$ & $12.43 \pm 6.29$ & 0.130 \\
\hline ACTH $(\mathrm{pg} / \mathrm{mL})$ & $38.12 \pm 23.96$ & $26.39 \pm 18.20$ & 0.095 \\
\hline PRL (ng/mL) & $21.34 \pm 40.13$ & $23.30 \pm 20.03$ & 0.796 \\
\hline $\mathrm{T}(\mathrm{ng} / \mathrm{mL})$ & $1.41 \pm 1.07$ & $1.75 \pm 1.62$ & 0.740 \\
\hline $\mathrm{P}(\mathrm{ng} / \mathrm{mL})$ & $1.30 \pm 2.78$ & $0.59 \pm 0.41$ & 0.237 \\
\hline $\mathrm{E} 2(\mathrm{pg} / \mathrm{mL})$ & $60.89 \pm 121.74$ & $103.63 \pm 263.72$ & 0.497 \\
\hline $\mathrm{LH}(\mathrm{IU} / \mathrm{L})$ & $10.82 \pm 10.74$ & $8.66 \pm 8.04$ & 0.303 \\
\hline FSH (IU/L) & $22.77 \pm 24.69$ & $21.52 \pm 22.67$ & 0.860 \\
\hline $\mathrm{Ca}(\mathrm{mmol} / \mathrm{L})$ & $2.36 \pm 0.18$ & $2.33 \pm 0.10$ & 0.403 \\
\hline $\mathrm{P}(\mathrm{mmol} / \mathrm{L})$ & $1.55 \pm 0.22$ & $1.26 \pm 0.13$ & $<0.001$ \\
\hline
\end{tabular}

BMI: body mass index; GH: growth hormone; IGF-1: insulin-like growth factor-1; T3: triiodothyronine; T4: thyroxine; TSH: thyroid-stimulating hormone; F: cortisol; ACTH: adrenocorticotropic hormone; PRL: prolactin; T: testosterone; P: progestogen; E2: oestradiol; LH: luteinizing hormone; FSH: folliclestimulating hormone; Ca: calcium; P: phosphorus.

TABLE 2: BMD of the GH-secreting pituitary adenoma group and the nonfunctioning adenoma group.

\begin{tabular}{lccc}
\hline & GH-secreting pituitary adenoma & Nonfunctioning pituitary adenoma & $p$ \\
\hline L1 & $1.107 \pm 0.187$ & $0.998 \pm 0.124$ & 0.006 \\
L1-Z & $0.433 \pm 1.414$ & $-0.186 \pm 1.116$ & 0.048 \\
L2 & $1.159 \pm 0.213$ & $1.067 \pm 0.132$ & 0.032 \\
L2-Z & $0.310 \pm 1.654$ & $-0.190 \pm 1.110$ & 0.141 \\
L3 & $1.228 \pm 0.220$ & $1.151 \pm 0.134$ & 0.078 \\
L3-Z & $0.562 \pm 1.630$ & $0.279 \pm 1.234$ & 0.419 \\
L4 & $1.200 \pm 0.227$ & $1.149 \pm 0.155$ & 0.271 \\
L4-Z & $0.344 \pm 1.720$ & $0.231 \pm 1.392$ & 0.767 \\
L1-L4 & $1.178 \pm 0.208$ & $1.097 \pm 0.125$ & 0.053 \\
L1-L4-Z & $0.418 \pm 1.583$ & $0.059 \pm 1.150$ & 0.282 \\
L2-L4 & $1.197 \pm 0.216$ & $1.124 \pm 0.131$ & 0.092 \\
L2-L4-Z & $0.408 \pm 1.642$ & $0.128 \pm 1.202$ & $0.906 \pm 0.133$ \\
Femoral neck & $1.034 \pm 0.161$ & $0.332 \pm 0.796$ & 0.420 \\
Femoral neck-Z & $0.882 \pm 1.120$ & $0.723 \pm 0.179$ & 0.01 \\
Ward triangle & $0.815 \pm 0.165$ & $-0.232 \pm 0.830$ & 0.022 \\
Ward triangle-Z & $-0.023 \pm 1.047$ & $0.746 \pm 0.111$ & 0.036 \\
Trochanter & $0.834 \pm 0.141$ & $-0.082 \pm 0.855$ & 0.366 \\
Trochanter-Z & $0.346 \pm 1.129$ & $1.118 \pm 0.136$ & 0.005 \\
Femoral shaft & $1.207 \pm 0.206$ & $0.940 \pm 0.119$ & 0.082 \\
Total femora & $1.034 \pm 0.164$ & $0.193 \pm 0.787$ & 0.037 \\
Total femora-Z & $0.531 \pm 1.196$ & & 0.008 \\
& & 0.168 \\
\hline
\end{tabular}

$0.032,0.001,0.036,0.005,0.037$, and 0.008 , respectively). There were no significant differences at other sites.

In terms of the $Z$ score, the BMD at L1 and the femoral neck were significantly elevated in patients with a $\mathrm{GH}$-secreting pituitary adenoma compared with controls ( $p=0.048$ and 0.022 , respectively). There were no significant differences at other sites.
3.2.2. Preoperative and Postoperative BMD in Patients with GH-Secreting Pituitary Adenomas. Thirteen patients with GH-secreting pituitary adenomas underwent re-examination of BMD 1 year after surgery, and the comparison of the preoperative and postoperative BMD is shown in Table 3. Compared with that of the preoperative $\mathrm{BMD}$, the $Z$ score of the postoperative BMD decreased significantly at L1, L2, L3, 
TABLE 3: Postoperative BMD $Z$ score changes in patients with acromegaly.

\begin{tabular}{lccrr}
\hline & Before surgery & After surgery & Control & P1 \\
\hline L1-Z & $0.600 \pm 1.134$ & $0.231 \pm 1.176$ & $-0.186 \pm 1.116$ & 0.037 \\
L2-Z & $0.546 \pm 1.385$ & $-0.046 \pm 1.212$ & $-0.190 \pm 1.110$ & 0.006 \\
L3-Z & $0.500 \pm 1.466$ & $0.115 \pm 1.289$ & $0.279 \pm 1.234$ & 0.029 \\
L4-Z & $0.477 \pm 1.705$ & $0.008 \pm 1.552$ & $0.231 \pm 1.392$ & 0.720 \\
L1-L4-Z & $0.539 \pm 1.433$ & $0.046 \pm 1.279$ & $0.059 \pm 1.150$ & 0.703 \\
L2-L4-Z & $0.508 \pm 1.532$ & $0.023 \pm 1.357$ & $0.128 \pm 1.202$ & 0.012 \\
Femoral neck-Z & $1.023 \pm 1.073$ & $1.269 \pm 1.085$ & $0.332 \pm 0.796$ & 0.012 \\
Ward triangle-Z & $0.231 \pm 1.154$ & $0.331 \pm 1.261$ & $-0.232 \pm 0.830$ & 0.019 \\
Trochanter-Z & $0.415 \pm 1.049$ & $0.477 \pm 1.042$ & $-0.082 \pm 0.855$ & 0.240 \\
Total hip-Z & $0.585 \pm 1.130$ & $0.777 \pm 1.173$ & $0.193 \pm 0.787$ & 0.619 \\
\hline
\end{tabular}

The patients with nonfunctioning pituitary adenoma were regarded as the control. P1: $p$ value of the comparison between preoperative and postoperative BMD; P2: $p$ value of the comparison between the postoperative and control group.

L4, L1-L4, and L2-L4 ( $p=0.037,0.006,0.029,0.031,0.012$, and 0.012 , respectively). The $Z$ score of postoperative BMD was significantly elevated in the femoral neck and total hip ( $p=0.019$ and 0.040 , respectively). There were no significant differences at other sites.

3.2.3. Analysis of the Potential Determinants of BMD. Pearson's correlation analysis was performed, and the results are shown in Table 4. A negative correlation was found between the disease duration and $Z$ score of the lumbar spine, including L1, L2, L3, and L4 sites (all $p<0.05$ ). In addition, the disease duration was also negatively correlated with the $Z$ score in the trochanter and total hip ( $p=0.048$ and 0.044 , respectively). IGF-1 burden was negatively correlated with the $Z$ score at L1 and L1-L4 ( $p=0.042$ and 0.048 , respectively). The PRL level was negatively correlated with the $Z$ score of the femoral neck and trochanter (all $p<0.05$ ). GH nadir, GH burden, T3, T4, TSH, PTH, ACTH, TC, TG, HDL, and LDL were also included in the correlation analysis and are not listed in Table 4 . There were no significant correlations found between these parameters and the BMD $Z$ score for any site (all $p>0.05$ ).

Multiple linear regression analysis showed that IGF-1 burden was negatively correlated with the preoperative BMD $Z$ score at L1 (Figure 1(a)) $(r=-0.398, p=0.007$, $R=0.631)$ and L1-L4 (Figure 1(b)) $(r=-0.387, p=0.01$, $R=0.619)$ in patients with $\mathrm{GH}$-secreting pituitary adenoma.

\section{Discussion}

Acromegalic osteopathy is a disease that seriously affects the quality of life of patients, and there has been no consistent conclusion regarding the effects of excess GH and IGF-1 on bone structure and bone metabolism, especially in terms of BMD changes. Our study revealed elevated BMD in patients with acromegaly, and the elevated lumbar-spine BMD recovered to a normal level after surgery. IGF-1 burden is concluded to be an independent risk factor for BMD reduction at L1. We adopted more sites for BMD measurement to obtain more comprehensive information than that yielded by the previous study. Patients with nonfunctioning pituitary adenomas were used as the control group, which could effectively reduce the interference of other potential factors.

The BMD in patients with active acromegaly was significantly elevated at L1, L2, femoral neck, Ward triangle, trochanter, femoral shaft, and total hip sites compared with that in patients with nonfunctioning pituitary adenomas. Conflicting results concerning the BMD changes in patients with acromegaly have remained over the past several decades. Previous studies by Zgliczynski et al. [5] and Kaji et al. [6] showed that BMD in the middle tibia, lumbar spine, femoral neck, and trochanter were significantly elevated in patients with acromegaly. Tuzcu et al. [7] reported that the BMD values in the lumbar spine and femoral neck of acromegaly patients were not significantly different from those in normal controls. Nevertheless, a few researchers have reported decreased BMD in patients with acromegaly [8]. On the one hand, patients with acromegaly are more likely to exhibit osteoarthritis in the spine and hip, resulting in structural changes. These changes may affect the measurement of BMD in the spine and hip [17]. On the other hand, the increase in bone area in patients with acromegaly may also lead to a decreased BMD, especially when using the DXA method [18]. The results of this study are consistent with those of most previous studies. We found that BMD in patients with acromegaly was elevated, and this change was likely to be the result of long-term chronic effects of high levels of GH and IGF-1. In previous investigations, the lumbar spine was regarded as a whole site, and the average BMD was considered. In our study, the BMD of the four lumbar vertebrae from L1 to L4 were evaluated in detail. In addition, the BMD at L1 and L2 in patients with acromegaly was elevated significantly. By comparing the $Z$ score, we were more convinced that the BMD at L1 was significantly elevated, while the BMD values at L3 and L4 were not changed.

Our study also found that after 1 year of surgery, the BMD $Z$ scores of the lumbar vertebrae in patients with acromegaly were significantly lower than the preoperative values, and there was no significant difference compared with the control group. It can be considered that the lumbarspine BMD recovers to a normal level after surgery. However, the BMD $Z$ score at the femoral neck continued to increase. Tamada et al. [19] showed that there was no 
TABLE 4: Correlation analysis of clinical data and the BMD $Z$ score.

\begin{tabular}{|c|c|c|c|c|c|c|c|c|c|c|c|}
\hline & & L1 & $\mathrm{L} 2$ & L3 & $\mathrm{L} 4$ & L1-L4 & L2-L4 & $\begin{array}{c}\text { Femoral } \\
\text { neck }\end{array}$ & $\begin{array}{l}\text { Ward } \\
\text { triangle }\end{array}$ & Trochanter & $\begin{array}{l}\text { Total } \\
\text { hip }\end{array}$ \\
\hline \multirow{2}{*}{ Age (years) } & $r$ & -0.110 & -0.125 & -0.156 & -0.125 & -0.123 & -0.131 & -0.017 & 0.068 & 0.056 & -0.039 \\
\hline & $p$ & 0.506 & 0.449 & 0.343 & 0.447 & 0.456 & 0.425 & 0.919 & 0.682 & 0.735 & 0.816 \\
\hline \multirow{2}{*}{ BMI $\left(\mathrm{kg} / \mathrm{m}^{2}\right)$} & $r$ & -0.049 & -0.048 & 0.069 & -0.034 & -0.014 & -0.023 & 0.082 & -0.102 & 0.054 & 0.235 \\
\hline & $p$ & 0.768 & 0.772 & 0.675 & 0.835 & 0.931 & 0.887 & 0.618 & 0.535 & 0.742 & 0.150 \\
\hline \multirow{2}{*}{ Disease duration (months) } & $R$ & $-0.389^{*}$ & $-0.370^{*}$ & $-0.371^{*}$ & $-0.371^{*}$ & $-0.413^{*}$ & $-0.393^{*}$ & -0.280 & -0.276 & $-0.319^{*}$ & $-0.324^{*}$ \\
\hline & $p$ & 0.014 & 0.021 & 0.020 & 0.020 & 0.009 & 0.013 & 0.084 & 0.088 & 0.048 & 0.044 \\
\hline \multirow{2}{*}{ Adenoma size (mm) } & $r$ & 0.070 & 0.183 & 0.144 & 0.147 & 0.139 & 0.155 & 0.102 & 0.120 & 0.104 & 0.114 \\
\hline & $p$ & 0.670 & 0.264 & 0.383 & 0.371 & 0.400 & 0.346 & 0.535 & 0.466 & 0.528 & 0.488 \\
\hline \multirow{2}{*}{$\mathrm{GH}(\mathrm{ng} / \mathrm{mL})$} & $r$ & 0.240 & 0.272 & 0.186 & 0.178 & 0.229 & 0.214 & 0.194 & 0.101 & 0.191 & 0.192 \\
\hline & $p$ & 0.140 & 0.094 & 0.257 & 0.277 & 0.161 & 0.190 & 0.236 & 0.541 & 0.245 & 0.241 \\
\hline \multirow{2}{*}{ IGF-1 (ng/mL) } & $r$ & 0.021 & 0.097 & 0.097 & 0.096 & 0.086 & 0.106 & 0.191 & 0.041 & 0.133 & 0.193 \\
\hline & $p$ & 0.897 & 0.556 & 0.559 & 0.563 & 0.603 & 0.522 & 0.244 & 0.802 & 0.421 & 0.240 \\
\hline \multirow{2}{*}{$\begin{array}{l}\text { IGF-1 burden (ng/ } \\
\mathrm{mL} * \text { month) }\end{array}$} & $r$ & $-0.326^{*}$ & -0.273 & -0.277 & -0.267 & $-0.319^{*}$ & -0.298 & -0.150 & -0.197 & -0.213 & -0.174 \\
\hline & $p$ & 0.042 & 0.093 & 0.088 & 0.100 & 0.048 & 0.066 & 0.362 & 0.230 & 0.194 & 0.291 \\
\hline \multirow{2}{*}{ FT3 (pg/mL) } & $r$ & -0.006 & -0.077 & -0.066 & -0.057 & -0.072 & -0.073 & -0.215 & -0.231 & -0.128 & -0.117 \\
\hline & $p$ & 0.970 & 0.643 & 0.692 & 0.729 & 0.665 & 0.659 & 0.188 & 0.157 & 0.436 & 0.477 \\
\hline \multirow{2}{*}{ FT4 $(\mathrm{pg} / \mathrm{mL})$} & $r$ & -0.195 & -0.176 & -0.081 & -0.096 & -0.124 & -0.118 & -0.100 & -0.057 & -0.095 & -0.107 \\
\hline & $p$ & 0.234 & 0.283 & 0.623 & 0.559 & 0.452 & 0.474 & 0.543 & 0.728 & 0.564 & 0.518 \\
\hline \multirow{2}{*}{ PRL (ng/mL) } & $r$ & -0.129 & -0.184 & -0.219 & -0.240 & -0.227 & -0.234 & $-0.329^{*}$ & -0.255 & $-0.361^{*}$ & -0.313 \\
\hline & $p$ & 0.435 & 0.263 & 0.180 & 0.142 & 0.164 & 0.151 & 0.041 & 0.117 & 0.024 & 0.053 \\
\hline \multirow{2}{*}{$\mathrm{Ca}(\mathrm{mmol} / \mathrm{L})$} & $r$ & 0.140 & 0.057 & 0.038 & 0.001 & 0.083 & 0.054 & 0.230 & 0.250 & 0.233 & 0.136 \\
\hline & $p$ & 0.396 & 0.730 & 0.819 & 0.995 & 0.614 & 0.746 & 0.159 & 0.124 & 0.154 & 0.411 \\
\hline \multirow{2}{*}{$\mathrm{P}(\mathrm{mmol} / \mathrm{L})$} & $r$ & 0.077 & 0.048 & 0.040 & -0.039 & 0.024 & 0.015 & -0.015 & -0.022 & -0.025 & 0.042 \\
\hline & $p$ & 0.641 & 0.771 & 0.808 & 0.812 & 0.883 & 0.929 & 0.929 & 0.897 & 0.878 & 0.801 \\
\hline
\end{tabular}

BMI: body mass index; GH: growth hormone; IGF-1: insulin-like growth factor-1; FT3: free triiodothyronine; FT4: free thyroxine; PRL: prolactin; Ca: calcium; P: phosphorus.

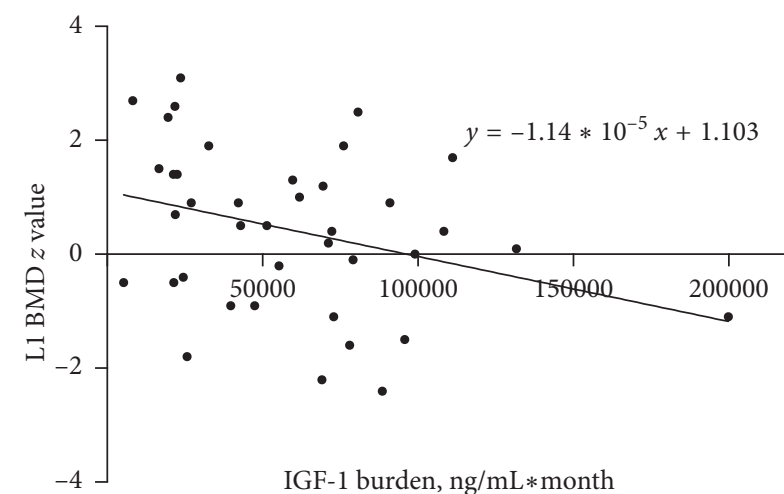

(a)

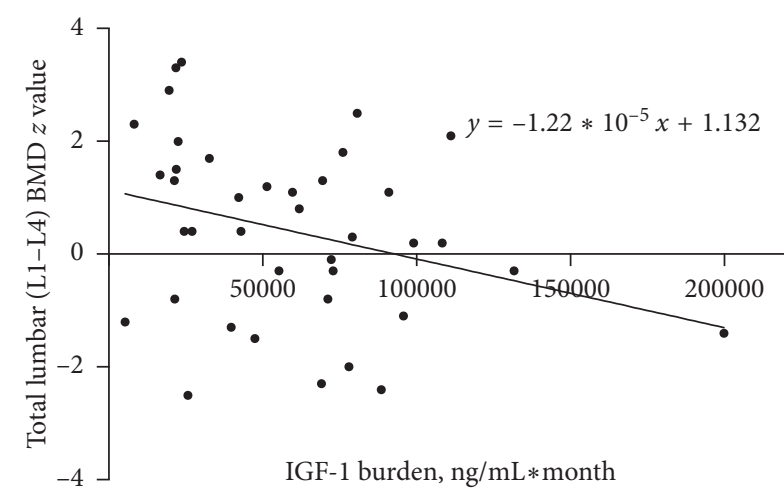

(b)

FIGURE 1: Correlation plot showing the correlations between IGF-1 burden and BMD Z scores: (a) a negative correlation was found between IGF-1 burden and the L1 BMD $Z$ score $(r=-0.326, p=0.042)$. (b) A negative correlation was found between IGF-1 burden and the total lumbar (L1-L4) BMD $Z$ score $(r=-0.319, p=0.048)$.

significant difference in BMD between the timepoint of 3 years after surgery and the preoperative period in patients with acromegaly. Mazziotti et al. [20] showed that there was no significant difference in lumbar-spine BMD between the timepoint of 3 years after surgery and the preoperative period, while the femoral neck BMD was significantly reduced. In both of the above studies, hormone replacement therapy was used in some patients to treat hypopituitarism before or after TSS, while GH replacement therapy may have promoted bone formation and increased BMD [21-23], which could have covered up the BMD decrease trend. In our study, there were no patients who underwent hormone replacement therapy; thus, we can exclude the effect of hormone replacement therapy on $\mathrm{BMD}$, which can reflect the changes in postoperative BMD more accurately. Since we do not have data from longer follow-up, the BMD changes over the subsequent few years are unclear. Further study will continue to focus on the postoperative BMD changes. Therefore, it can be concluded that the elevated lumbarspine BMD in patients with acromegaly recovered to normal levels after surgery when the GH and IGF-1 levels were controlled. 
In addition, the results of our study are different from those of previous studies. The postoperative BMD $Z$ score decreased at the lumbar vertebrae and was elevated at the femoral neck. The difference in structure and mechanics can account for this difference. In terms of structure, the lumbar spine is rich in trabecular bone, while cortical bone is prevalent at the femoral neck [24]. BMD at trabecular bone has been reported to decrease in patients with acromegaly [8]. Other studies have shown that excessive GH and IGF-1 have a deleterious effect on trabecular bone, while $\mathrm{GH}$-induced periosteal ossification increases the BMD in cortical bone [21, 25]. Therefore, we believe that the different effects of excessive GH and IGF-1 on cortical and trabecular bones result in differences in the trend of BMD between the lumbar spine and the femoral neck.

We found that the disease duration and IGF-1 burden were negatively correlated with $\mathrm{BMD} Z$ score. The multiple linear regression also showed that IGF-1 burden is an independent risk factor for reduced preoperative L1 BMD $Z$ score. Previous studies have shown a negative correlation between BMD and disease duration [8], and lumbar-spine $\mathrm{BMD}$ is positively correlated with IGF-1 levels [7]. Some other studies have shown that lumbar-spine BMD is negatively correlated with the duration of hypogonadism, positively correlated with the duration of acromegaly, but not correlated with the IGF-1 level. Femoral neck BMD is positively correlated with the IGF-1 level and not correlated with the duration of hypogonadism and the duration of acromegaly [26]. By analysing previous studies, we believe that the increase in BMD may be due to the long-term changes in bone structure caused by excess GH and IGF-1. Our study showed that IGF-1 burden $($ IGF- 1 level $\times$ disease duration) was negatively correlated with BMD $Z$ score at the lumbar spine. Therefore, we concluded that IGF-1 burden was an excellent indicator of BMD. However, we only came to this conclusion for the BMD $Z$ score at L1, and further research is waiting to be verified by using more samples and by adopting additional measurement sites.

The serum phosphorus level was significantly increased in patients with acromegaly in our study. Hyperphosphatemia is frequently observed in patients with acromegaly [27-29]. The elevated phosphorus level was slightly higher than the upper normal range. The correlation analysis showed that phosphorus level was not significantly correlated with preoperative BMD in patients with GH-secreting pituitary adenoma. Therefore, the effect of a higher phosphorus level on elevated $\mathrm{BMD}$ in patients with $\mathrm{GH}$-secreting pituitary adenoma may not be dominant in our study. In addition, the FT3 and FT4 levels were significantly higher in patients with acromegaly than in patients with nonfunctioning pituitary adenomas in our study. Excessive GH has been reported to have effects on thyroid function, resulting in changes in thyroid hormone [30]. Pearson's correlation analysis showed that the thyroid hormone levels were not significantly correlated with BMD in patients with $\mathrm{GH}$-secreting pituitary adenoma. The elevated FT3 and FT4 levels were still within the upper normal range. The T3 and T4 levels were also within normal range. A previous study on postmenopausal women showed that elevated thyroid hormones levels within the upper normal range were associated with decreased BMD [31]. Therefore, we believe that the variation in thyroid hormones levels contributed little to the increase in the BMD of patients with GH-secreting pituitary adenoma.

Our investigation has its limitations. First, this is a retrospective study, and the randomness was not fully satisfied. Therefore, the conclusions drawn from the study population did not fully represent the overall situation of patients with pituitary GH adenomas. Second is the limitation of the DXA method for BMD measurement. This method cannot well distinguish cortical bone and trabecular bone. As mentioned above, there are differences in the effects of excessive GH and IGF-1 on cortical bone and trabecular bone. Therefore, the results did not fully reflect the detailed changes in BMD. In addition, the DXA method cannot detect changes in bone microarchitecture and is easily interfered with by factors such as periosteal ossification and osteoarthritis. In contrast, qCT can measure BMD in three dimensions and can also distinguish between cortical bone and trabecular bones [32]. However, considering radiation exposure and medical expenses, the DXA method is still a good tool for observing the general trend of BMD change. Finally, the size of the study population was very limited. Due to medical expenses, traffic problems, and concerns about radiation exposure, many patients did not have regular follow-up and BMD re-examinations.

Despite the above limitations, our study can help us better understand the pathophysiological influence of excessive GH and IGF-1 on bone metabolism. As the disease duration and IGF-1 burden were negatively correlated with the preoperative BMD in patients with $\mathrm{GH}$-secreting pituitary adenoma, clinical intervention on BMD should be conducted in the acromegalic patients who have higher levels of IGF-1 or longer disease duration. It is hypothesized that the BMD may be a biomarker of disease activity in patients with acromegalic osteopathy, but further studies are needed to confirm this hypothesis. Furthermore, BMD may be considered a routine examination in acromegaly patients.

\section{Conclusions}

In conclusion, the preoperative BMD in patients with acromegaly is significantly elevated at L1, L2, femoral neck, Ward triangle, trochanter, femoral shaft, and total hip sites. The lumbar-spine BMD recovered to normal levels after successful surgery when GH and IGF-1 levels were controlled. Preoperative lumbar-spine BMD $Z$ score was negatively correlated with disease duration and IGF-1 burden, and IGF-1 burden was an independent risk factor for preoperative BMD $Z$ score decrease at L1 in patients with acromegaly.

\section{Data Availability}

All data included in this study are available upon request by contact with the corresponding author.

\section{Conflicts of Interest}

The authors declare that there are no conflicts of interest regarding the publication of this paper. 


\section{Authors' Contributions}

Li'nan Qin and Xiaopeng Guo contributed equally to this study. Li'nan Qin, Xiaopeng Guo, and Bing Xing designed the study. Xiaopeng Guo and Bing Xing monitored all experiments. Li'nan Qin, Xiaopeng Guo, Lu Gao, Chenzhe Feng, Kan Deng, Wei Lian, and Zihao Wang performed the experiments and analysed the data. Li'nan Qin and Xiaopeng Guo wrote the manuscript, and Bing Xing revised the manuscript.

\section{References}

[1] R. Bouillon, "Growth hormone and bone," Hormone Research, vol. 36, no. 1, pp. 49-55, 1991.

[2] G. Padova, G. Borzi, L. Incorvaia et al., "Prevalence of osteoporosis and vertebral fractures in acromegalic patients," Clinical Cases in Mineral and Bone Metabolism, vol. 8, no. 3, pp. 37-43, 2011.

[3] L. Katznelson, E. R. Laws Jr., S. Melmed et al., "Acromegaly: an endocrine society clinical practice guideline," The Journal of Clinical Endocrinology \& Metabolism, vol. 99, no. 11, pp. 3933-3951, 2014.

[4] Y. Kasukawa, N. Miyakoshi, and S. Mohan, "The anabolic effects of GH/IGF system on bone," Current Pharmaceutical Design, vol. 10, no. 21, pp. 2577-2592, 2004.

[5] W. Zgliczynski, M. Kochman, W. Misiorowski, and P. Zdunowski, "In acromegaly, increased bone mineral density (BMD) is determined by GH-excess, gonadal function and gender," Neuro Enocrinology Letters, vol. 28, no. 5, pp. 621-628, 2007.

[6] H. Kaji, T. Sugimoto, D. Nakaoka et al., "Bone metabolism and body composition in Japanese patients with active acromegaly," Clinical Endocrinology, vol. 55, no. 2, pp. 175-181, 2001.

[7] S. Tuzcu, Ş. A. Durmaz, A. Carlıoğlu et al., "The effects of high serum growth hormone and IGF-1 levels on bone mineral density in acromegaly," Zeitschrift für Rheumatologie, vol. 76, no. 8, pp. 716-722, 2016.

[8] E. Valassi, I. Crespo, J. Malouf et al., "Reduction of trabecular and cortical volumetric bone mineral density at the proximal femur in patients with acromegaly," European Journal of Endocrinology, vol. 174, no. 2, pp. 107-114, 2016.

[9] N. A. Tritos and A. Klibanski, "Effects of growth hormone on bone," Progress in Molecular Biology and Translational Science, vol. 138, pp. 193-211, 2016.

[10] J. A. Langlois, C. J. Rosen, M. Visser et al., “Association between insulin-like growth factor I and bone mineral density in older women and men: the framingham heart study," The Journal of Clinical Endocrinology \& Metabolism, vol. 83, no. 12, pp. 4257-4262, 1998.

[11] E. Barrett-Connor and D. Goodman-Gruen, "Gender differences in insulin-like growth factor and bone mineral density association in old age: the Rancho Bernardo study," Journal of Bone and Mineral Research, vol. 13, no. 8, pp. 1343-1349, 1998.

[12] J. Janssen, H. Burger, R. Stolk et al., "Gender-specific relationship between serum free and total IGF-I and bone mineral density in elderly men and women," European Journal of Endocrinology, vol. 138, no. 6, pp. 627-632, 1998.

[13] D. Bikle, S. Majumdar, A. Laib et al., "The skeletal structure of insulin-like growth factor I-deficient mice," Journal of Bone and Mineral Research, vol. 16, no. 12, pp. 2320-2329, 2001.
[14] M. E. Molitch, "Diagnosis and treatment of pituitary adenomas: a review," JAMA, vol. 317, no. 5, pp. 516-524, 2017.

[15] A. Giustina, P. Chanson, M. D. Bronstein et al., "A consensus on criteria for cure of acromegaly," The Journal of Clinical Endocrinology \& Metabolism, vol. 95, no. 7, pp. 3141-3148, 2010.

[16] X. Guo, L. Gao, S. Zhang et al., "Cardiovascular system changes and related risk factors in acromegaly patients: a casecontrol study," International Journal of Endocrinology, vol. 2015, Article ID 573643, 7 pages, 2015.

[17] M. J. E. Wassenaar, N. R. Biermasz, N. van Duinen et al., "High prevalence of arthropathy, according to the definitions of radiological and clinical osteoarthritis, in patients with long-term cure of acromegaly: a case-control study," European Journal of Endocrinology, vol. 160, no. 3, pp. 357-365, 2009.

[18] T. Ueland, S. L. Fougner, K. Godang, T. Schreiner, and J. Bollerslev, "Serum GH and IGF-I are significant determinants of bone turnover but not bone mineral density in active acromegaly: a prospective study of more than 70 consecutive patients," European Journal of Endocrinology, vol. 155, no. 5, pp. 709-715, 2006.

[19] D. Tamada, T. Kitamura, M. Takahara et al., "Postoperative changes in bone metabolism and bone mineral density in Japanese patients with acromegaly: a 3-year prospective study," Endocrine Journal, vol. 62, no. 11, pp. 1031-1036, 2015.

[20] G. Mazziotti, A. Bianchi, T. Porcelli et al., "Vertebral fractures in patients with acromegaly: a 3-year prospective study," The Journal of Clinical Endocrinology \& Metabolism, vol. 98, no. 8, pp. 3402-3410, 2013.

[21] A. Giustina, G. Mazziotti, and E. Canalis, "Growth hormone, insulin-like growth factors, and the skeleton," Endocrine Reviews, vol. 29, no. 5, pp. 535-559, 2008.

[22] G. Johannsson, T. Rosen, I. Bosaeus, L. Sjostrom, and B. A. Bengtsson, "Two years of growth hormone $(\mathrm{GH})$ treatment increases bone mineral content and density in hypopituitary patients with adult-onset GH deficiency," Journal of Clinical Endocrinology \& Metabolism, vol. 81, no. 8, pp. 2865-2873, 1996.

[23] C. Ohlsson, B. A. Bengtsson, O. G. Isaksson, T. T. Andreassen, and M. C. Slootweg, "Growth hormone and bone," Endocrine Reviews, vol. 19, no. 1, pp. 55-79, 1998.

[24] M. Madeira, L. V. Neto, F. de Paula Paranhos Neto et al., "Acromegaly has a negative influence on trabecular bone, but not on cortical bone, as assessed by high-resolution peripheral quantitative computed tomography," The Journal of Clinical Endocrinology \& Metabolism, vol. 98, no. 4, pp. 1734-1741, 2013.

[25] K. M. J. A. Claessen, G. Mazziotti, N. R. Biermasz, and A. Giustina, "Bone and joint disorders in acromegaly," Neuroendocrinology, vol. 103, no. 1, pp. 86-95, 2016.

[26] A. Scillitani, C. Battista, I. Chiodini et al., "Bone mineral density in acromegaly: the effect of gender, disease activity and gonadal status," Clinical Endocrinology, vol. 58, no. 6, pp. 725-731, 2003.

[27] S. Takamoto, H. Tsuchiya, T. Onishi et al., "Changes in calcium homeostasis in acromegaly treated by pituitary adenomectomy," The Journal of Clinical Endocrinology \& Metabolism, vol. 61, no. 1, pp. 7-11, 1985.

[28] J. Halse and H. N. Haugen, "Calcium and phosphate metabolism in acromegaly," Acta Endocrinologica, vol. 94, no. 4, pp. 459-467, 1980.

[29] G. Y. Yalin, S. Tanrikulu, N. Gul, A. K. Uzum, F. Aral, and R. Tanakol, "Utility of baseline serum phosphorus levels for 
predicting remission in acromegaly patients," Journal of Endocrinological Investigation, vol. 40, no. 8, pp. 867-874, 2017.

[30] M. Losa, M. Scavini, E. Gatti et al., "Long-term effects of growth hormone replacement therapy on thyroid function in adults with growth hormone deficiency," Thyroid, vol. 18, no. 12 , pp. $1249-1254,2008$.

[31] E. Murphy, C. C. Glüer, D. M. Reid et al., "Thyroid function within the upper normal range is associated with reduced bone mineral density and an increased risk of nonvertebral fractures in healthy euthyroid postmenopausal women," The Journal of Clinical Endocrinology \& Metabolism, vol. 95, no. 7, pp. 3173-3181, 2010.

[32] J. R. Anthony and A. G. Ioachimescu, "Acromegaly and bone disease," Current Opinion in Endocrinology \& Diabetes and Obesity, vol. 21, no. 6, pp. 476-482, 2014. 


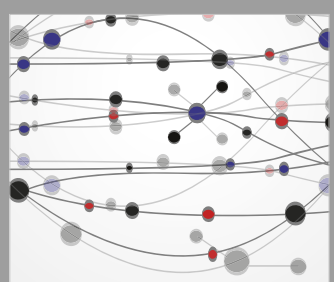

The Scientific World Journal
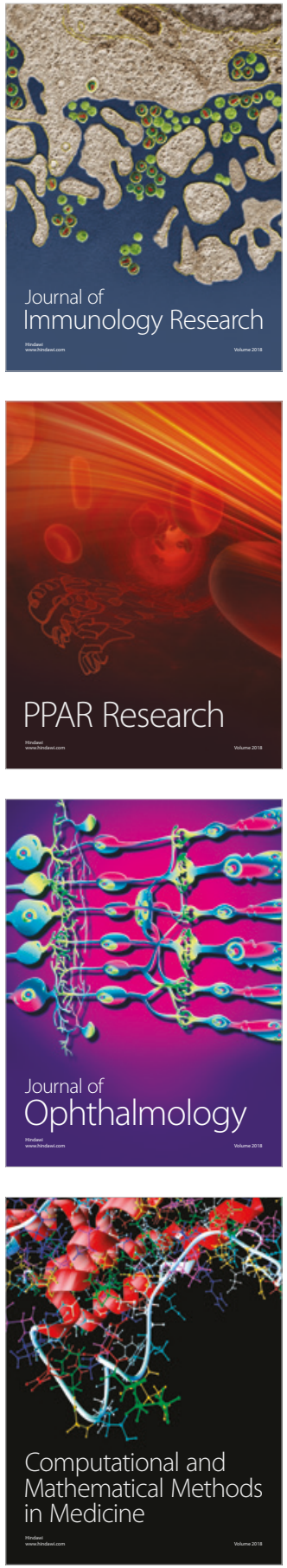

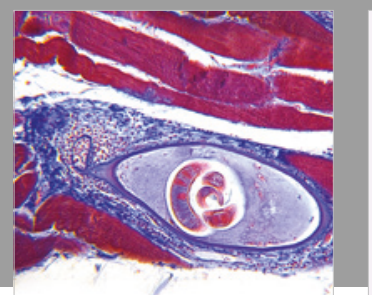

Gastroenterology Research and Practice

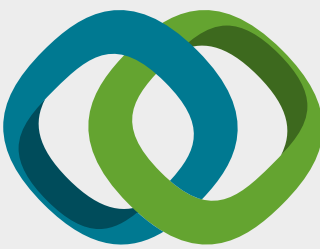

\section{Hindawi}

Submit your manuscripts at

www.hindawi.com
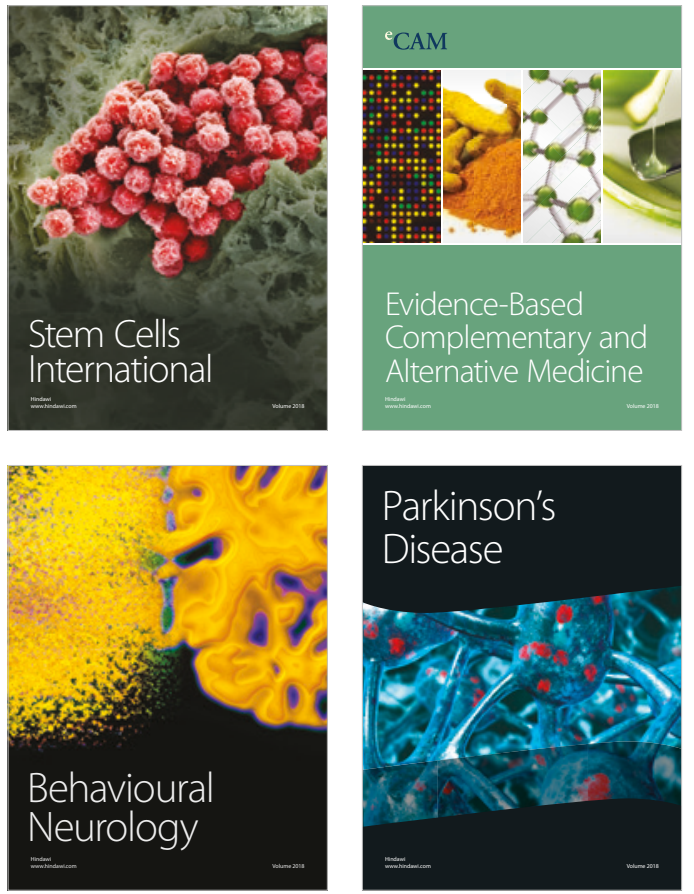

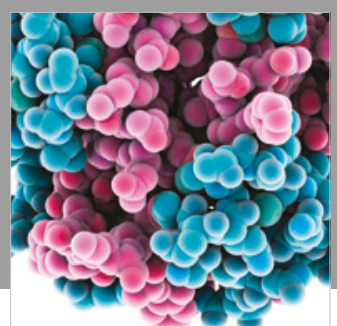

ournal of

Diabetes Research

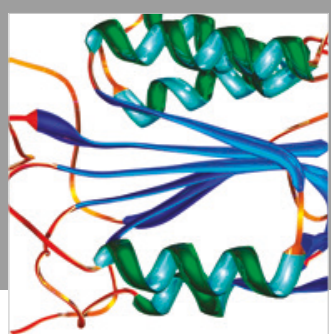

Disease Markers
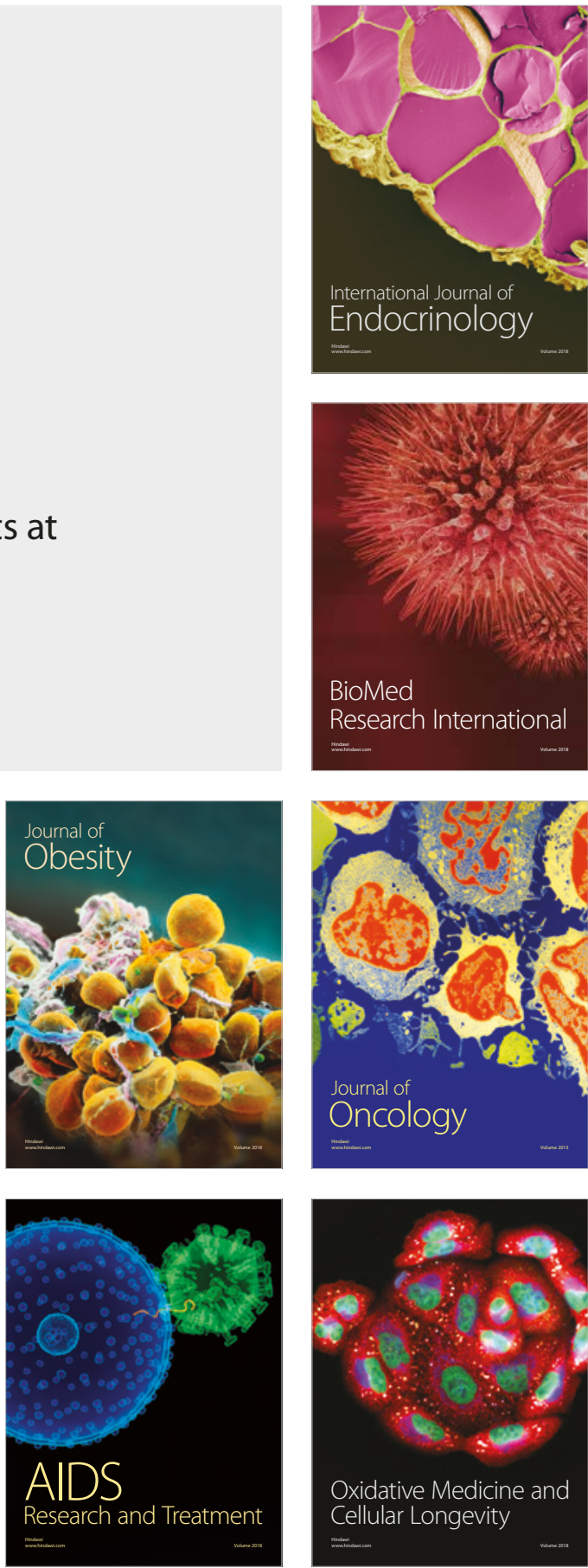